\title{
Location of Myocardium at Risk in Comparison between Single Photon Emission Computed Tomography, Magnetic Resonance Imaging and Electrocardiography
}

\author{
JFA Ubachs ${ }^{1}$, APM Gorgels ${ }^{1}$, E Hedstrom ${ }^{2}$, H Arheden ${ }^{2}$, RH Selvester ${ }^{3}$, \\ SAM Knippenberg ${ }^{1}$, GS Wagner ${ }^{4}$, H Engblom ${ }^{2}$ \\ ${ }^{1}$ University Hospital Maastricht, Maastricht, The Netherlands \\ ${ }^{2}$ Lund University Hospital, Lund, Sweden \\ ${ }^{3}$ Memorial Heart Institute of Long Beach, CA, USA \\ ${ }^{4}$ Duke University Medical Center, Durham, NC, USA
}

\begin{abstract}
The aim of the present study was to test the hypothesis that perfusion SPECT, estimation of regional wall thickening using MRI and the distribution of ST changes on the admission ECG display similar localization of the MaR in patients with reperfused first-time MI.

Eleven patients with first-time MI with ST-elevation underwent percutaneous coronary intervention (PCI), SPECT imaging within 3 hours of the PCI and Cardiac MRI the day after admission. All images were presented in polar plots and compared with polar plots generated from the initial ECG's, based upon ST-changes.

Comparison of the displays reveals similarity of location of myocardium at risk by all three methods in $73 \%$ of the patients, between SPECT and ECG in $91 \%$ of the patients, and between SPECT and wall thickening by MRI in $73 \%$ of the patient.
\end{abstract}

\section{Introduction}

The prognosis in patients with acute coronary syndrome is directly related to myocardial infarct (MI) size. The major determinants of MI size is the myocardium at risk (MaR) during the acute coronary occlusion in relation to the duration of persistent occlusion. The goal of early reperfusion therapy is to limit the amount of this region that is infarcted, to achieve maximally preserved left ventricular function and to decrease occurrence of either heart failure or sudden death.

To assess the efficiency of reperfusion therapy, it is necessary to determine how much myocardium is salvaged by measuring the final infarct size in relation to the initial MaR.

The most widely used clinical method to diagnose acute coronary occlusion is by observing ST segments deviation on the 12-lead electrocardiogram (ECG). ST segment based methods have previously been shown to provide accurate diagnosis and localization of $\mathrm{MaR}$ in patients with coronary occlusion. ${ }^{[1,2]}$

The most widely practiced technique for directly measuring MaR is currently perfusion SPECT. This approach requires injection of a technetium based tracer before opening of the artery and access to a gamma camera at the time when the patient presents at the hospital.

After opening of an occluded coronary artery, myocardial dysfunction persists in the MaR over a variable period of time depending on the severity of the ischemia and success of the reperfusion therapy. Therefore, clinical methods for detecting areas of dysfunctional myocardium soon after an episode of coronary occlusion might provide an estimate of MaR. Magnetic resonance imaging (MRI) is an excellent method for assessing the regional wall thickening during the cardiac cycle. ${ }^{[3]}$ Thus, MRI can be used to assess the dysfunctional region resulting from an episode of coronary occlusion.

The aim of the present study was to test the hypothesis that perfusion SPECT, estimation of regional wall thickening using MRI and the distribution of ST changes on the admission ECG display similar localization of the $\mathrm{MaR}$ in patients with reperfused first-time MI.

\section{Methods}

\subsection{Study population and design}

The study was approved by the local ethics committee and all patients gave their written informed consent to participate. Eleven patients with symptoms and ST segment changes suggestive of acute coronary occlusion were included in the study. Patients with a history of prior infarction, angiographic signs of collateral flow supplying the occluded vessel, and creatine kinase MB or 
troponin $\mathrm{T}$ release prior to intervention were excluded. All patients had a complete coronary occlusion and were treated by primary percutaneous coronary intervention (PPCI) with stenting, resulting in TIMI grade 3 flow. A glycoprotein receptor IIb/IIIa inhibitor was administered in conjunction with the PPCI. Immediately prior to PPCI,

${ }^{99 \mathrm{~m}} \mathrm{Tc}$ tetrofosmin was injected and myocardial perfusion SPECT was performed within 3 hours after PPCI for determination of MaR. Cardiac MRI was undertaken the day after admission. Patients with contraindication for MRI was also excluded.

\subsection{Electrocardiographic recordings}

Standard 12-lead ECG was recorded at the time of patient. The admission ECG was used to designate the location of the acute ischemia based on ST-deviation patterns according to methods described by Aldrich et al. $^{[1]}$

\subsection{Risk area assessment}

\subsubsection{Electrocardiographic}

The Selvester QRS scoring system was developed to estimate the global size of MI in the LV using the standard 12 lead ECG. ${ }^{[4]}$ The Selvester QRS scoring system also provides an estimate of the distribution of the MI into 12 left ventricular segments by considering the leads in which QRS waveforms meet the criteria. In the present study, the leads with ST segment deviation were used to distribute the ECG estimated risk regions by the same 12 segment model used for the Selvester QRS scoring system. Two research physicians, blinded to each others results, estimated the location of the MaR based on the ST-changes found in the initial ECG, using the distribution of the lead involvement for each segment and the direction of the vector.

\subsubsection{MRI imaging and analysis}

Magnetic resonance imaging was performed on either of two 1.5 T systems: Magnetom Vision (Siemens, Erlangen, Germany) with a CP body array coil, or Philips Intera CV (Philips, Best, the Netherlands) with a cardiac synergy coil. All subjects were placed in supine position. Short- and long-axis images were acquired, covering the left ventricle (LV) to assess systolic wall thickening. ${ }^{[5]}$ The wall thickening was calculated as the percent change in radial wall thickness between end-diastole and endsystole after manually tracing of the endocardial and epicardial borders. For the wall thickening analysis the papillary muscles were excluded from the myocardium.

\subsubsection{Myocardial perfusion SPECT}

Patients were injected with 500-700 $\mathrm{MBq}{ }^{99 \mathrm{~m}} \mathrm{Tc}$ tetrofosmin, depending on bodyweight. Myocardial perfusion SPECT was performed according to the standard clinical protocol at rest, using a dual head camera. The patient was placed in supine position and imaged in steps of 5.6 degrees using a $64 \times 64$ matrix with a pixel size of $5.02 \mathrm{~mm}$. Image acquisition time was approximately 25 minutes. Short- and long-axis images, covering the left ventricle, gated to ECG, were then reconstructed.

\subsection{Polar plot representation}

Polar plots were generated using the quantitative data from perfusion SPECT and MRI wall thickening analysis. All data were treated as polar coordinates and subsequently converted to Cartesian coordinates for graphing as polar plots. The quantitative values of perfusion SPECT and MRI wall thickening were represented in each polar plot by a scale ranging from white or warm colors (most normal) to black or cold color (most abnormal). The perfusion color scale was set to range between 0 and $100 \%$ of maximum $\mathrm{LV}$ perfusion on a per-patient basis. The wall thickening color scale was set to range between 0 and $100 \%$ of wall thickening on a per-segment basis. ${ }^{[3]}$

In order to compare the ECG findings with the imaging polar views, the ECG findings were also viewed as polar plots. The mercator views were divided into 12 segments and manually adjusted so that each segment had the same shape and size, containing the amount of MaR estimated by two research physicians blinded to each other. These adjusted mercator views were then converted into polar plots using specific graphic applications (Corel, Ottawa, Canada), for comparison with the perfusion SPECT and MRI wall thickening polar plots.

Describe your methods here.

\section{Results}

All eleven patients included were male and had a mean age of 66. Fifty-five percent (6/11) of the patients had an occlusion in the left anterior descendent artery (LAD), $36 \%(4 / 11)$ in the right coronary artery (RCA), and $9 \%$ $(1 / 11)$ in the left circumflex artery (LCX). Visual comparisons, by the 3 methods of the locations of the risk regions of each patient, grouped according to occlusion location, are presented in figures $1-3$ by polar plots.

In $67 \%(4 / 6)$ of the patients with LAD occlusion, all three methods showed similar locations of the MaR, involving the anteroseptal and anterosuperior quadrants of the left ventricle. In patient 2, the ECG shows that all the myocardium at risk is located in the anterosuperior quadrant with no involvement of the anteroseptal quadrant when compared with the SPECT data. The ECG 
from patient 2 also showed that the extent of MaR did not covering all the segments in the anterosuperior quadrant.
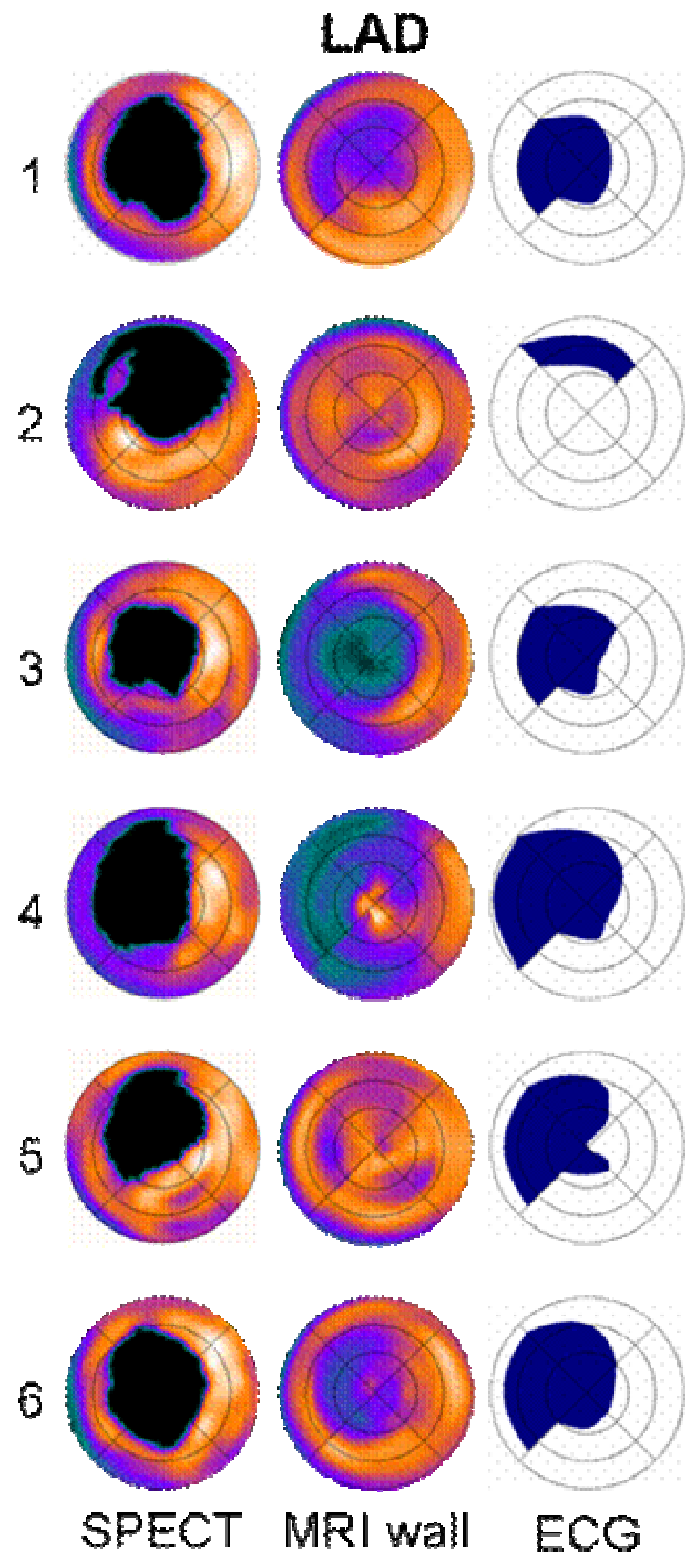

\section{MRI wal thickening}

Figure 1: Polar plots generated from rest ${ }^{99 \mathrm{~m}} \mathrm{Tc}$-tetrofosmin SPECT perfusion, MRI wall thickening and ECG for patients with an LAD occlusion. The perfusion color scale: warm colors indicate good perfusion and cold colors indicate less or absence of perfusion.

The wall thickening color scale: Warm colors represent good wall thickening and cold colors indicate hypokinesia or akinesia.

Patient 2 had no region with decreased wall thickening with MRI. This patient showed no infarction by contrastenhanced MRI, and had low peaks of creatine kinase and troponin after PPCI, indicating a high degree of myocardial salvage. In patient 5 , there was no defined region with decreased wall thickening by MRI.

RCA
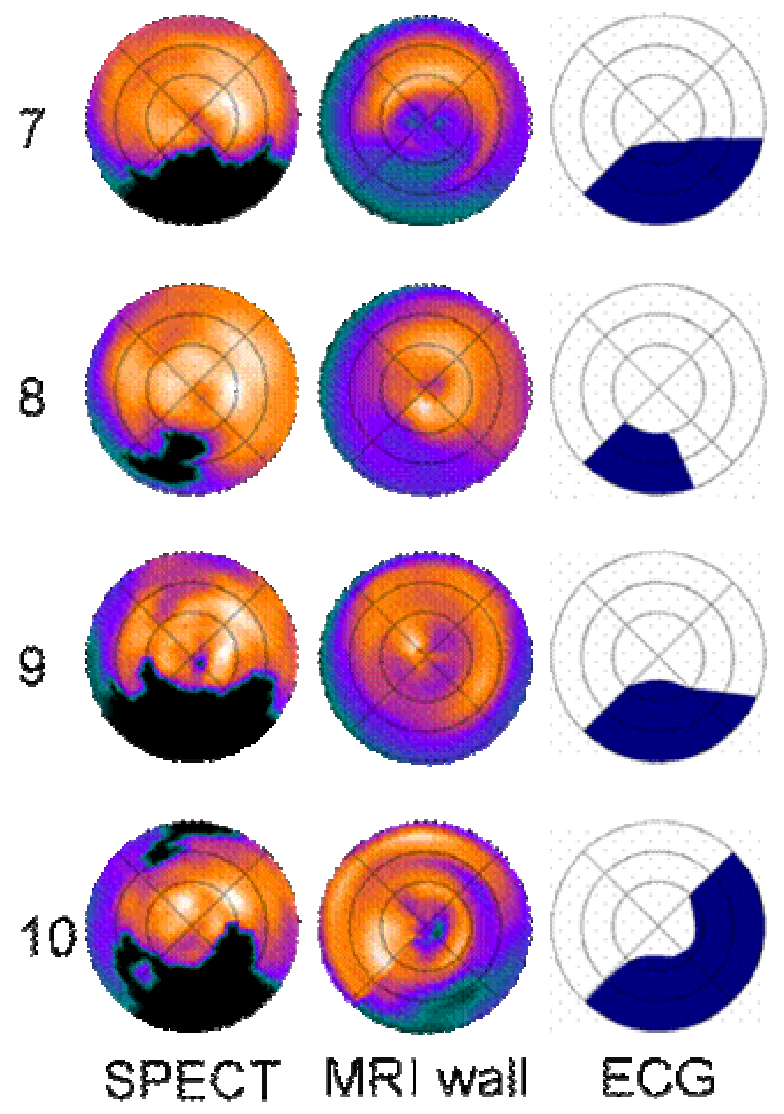

ECG thickening

Figure 2: Polar plots generated from rest ${ }^{99 \mathrm{~m}} \mathrm{Tc}$-tetrofosmin SPECT perfusion, MRI wall thickening and ECG for all patients with an RCA occlusion.

The location of the MaR was similar by all three methods in $50 \%(2 / 4)$ of the patients with RCA occlusion. The MaR in these patients included either the inferior quadrant or inferior and posterolateral quadrant. The wall thickening by MRI for patient 9 showed no dysfunction in the middle segment of the posterolateral quadrant. In patient 10 , the location of the MaR showed a good visual agreement comparing MRI wall thickening and ECG with perfusion SPECT. However in patient 10, the MaR extended more in the posterolateral quadrant by ECG and by MRI compared to perfusion SPECT.

The patient with occlusion of the LCX had MaR both in the inferior and the posterolateral quadrant (Figure 3). 
The angiography revealed left dominance, looking at the posterior descending artery originating from LCX.

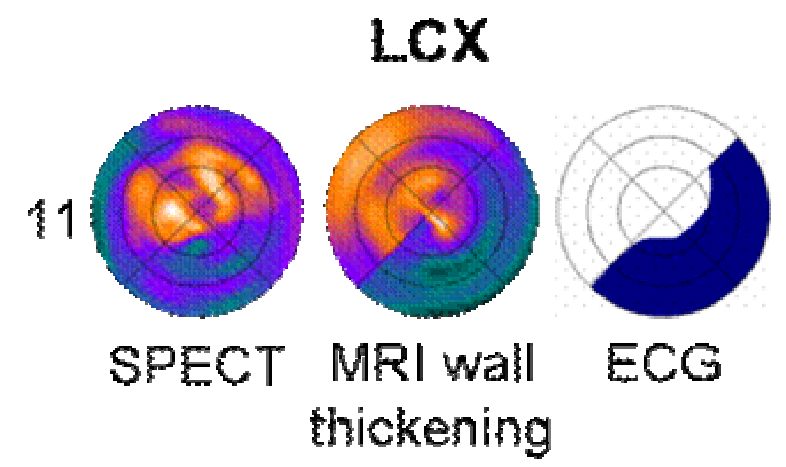

Figure 3: Polar plots generated from rest ${ }^{99 \mathrm{~m}} \mathrm{Tc}$-tetrofosmin SPECT perfusion, MRI wall thickening and ECG for all patients with an LCX occlusion.

\section{Discussion and conclusions}

To our knowledge, the present study is the first to examine the method for translating outputs from various clinical tests into comparable displays and examine the visualized estimates of MaR.

This study shows not only the visual correlation between different estimates of myocardial infarction but also shows the feasibility of the quantitative polar representation of myocardial perfusion, function and ECG as a uniform method that can potentially be used clinically to help clinicians make earlier decision about myocardium at risk for infarction.

ST segment elevation is the accepted clinical indicator that coronary occlusion has produced "myocardium at risk" for infarction. The direction of the ST elevation has also been proven to indicate the location of the LV myocardial region at risk. Previous studies have even suggested that the degree of initial ST segment elevation can quantify the extent of jeopardized myocardium. ${ }^{[6]}$ The present study shows that there is a good visual representation of the direction of the ST-deviations in the initial ECG with myocardial perfusion SPECT for estimating location and extent of the myocardium at risk. Currently, decisions are made by observing the extent of ST elevation. In the future, clinicians may be able to visualize spatial involvement when looking at STsegment changes or manufacturers may incorporate this method into the display of ECG recordings.

Holman et al. ${ }^{[7]}$ showed that MRI wall thickening could be used to quantify left ventricular dysfunction. Our study shows that MRI wall thickening can be used in humans to localize and estimate the extent of the myocardium at risk.

Polar representation of localization of myocardium at risk by SPECT perfusion, MRI wall thickening and ECG
ST-deviation is feasible. These results suggest that this method can be of clinical value to aid in the assessment of patients with myocardial infarction. Comparison of the displays reveals similarity of location of myocardium at risk by all three methods in $73 \%$ of the patients, between SPECT and ECG in $91 \%$ of the patients, and between SPECT and wall thickening by MRI in $73 \%$ of the patients.

\section{References}

[1] Aldrich HR, Hindman NB, et al. Identification of the optimal electrocardiographic leads for detecting acute epicardial injury in acute myocardial infarction. Am J Cardiol 1987;59:20-23

[2] Aldrich HR, Corsa AT. ST deviation in an acute MI: a predictive formula, Engineering Foundation Press 1986, 243-248.

[3] Cain PA, Ugander M. Quantitative polar representation of left ventricular myocardial perfusion, function and viability using SPECT and cardiac magnetic resonance: initial results. Clin Physiol Funct Imaging 2005;25:215-222

[4] Selvester RH, Wagner GS. The Selvester QRS scoring system for estimating myocardial infarct size: the development and application of the system. Arch Intern Med 1985;145:1877-1881.

[5] Engblom H, Hedstrom E. Determination of the left ventricular long-axis orientation from a single short-axis MR image: relation to BMI and age. Clin Physiol Funct Imaging 2004;24:310-315.

[6] Christian TF, Gibbons RJ. Estimates of myocardium at risk and collateral flow in acute myocardial infarction using electrocardiographic indexes with comparison to radionuclide and angiographic measures. J Am Coll Cardiol 1995;26:388-93.

[7] Holman ER, Buller VGM. Detection and quantification of dysfunctional myocardium by magnetic resonance imaging. A new three-dimensional method for quantitative wall-thickening analysis. Circulation 1997;95:924-931.

Address for correspondence

Galen S. Wagner, MD

Duke University Medical Center

2400 Pratt Street

North Pavilion, Terrace Level, Rm 0311

Durham, North Carolina 27705 